Kompass

Pneumologie

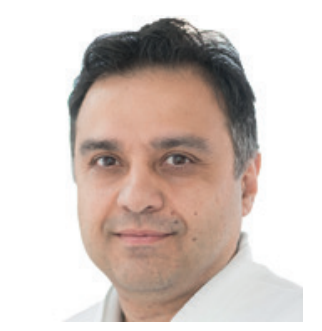

Fotios Drakopanagiotakis

Medizinische Klinik V, Pneumologie, Klinikum Fulda, Universitätsmedizin Marburg - Campus Fulda, Fulda,

Deutschland

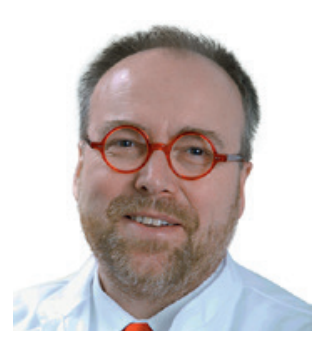

\section{Andreas Günther}

Pneumologische Klinik \& Zentrum für Innere Medizin, Agaplesion Krankenhaus Mittelhessen, Medizinische Klinik II, Universitätsklinikum Gießen, Gießen, Deutschland

\title{
Respiratorische Viren, COVID und Lungenfibrose - alles wie beim ARDS oder doch anders?
}

Das neuartige SARS-CoV-2-Coronavirus, das für die COVID-19-Krankheit verantwortlich ist, wurde erstmals im Dezember 2019 in Wuhan, China, gemeldet. Mit Millionen von bestätigten Fällen weltweit wächst die Besorgnis über das Potenzial einer Coronavirus-Infektion, chronische Atemwegssymptome oder fibrotische Erkrankungen bei genesenen Personen zu verursachen [1]. Bisher sind sieben Arten von Coronaviren identifiziert worden, die den Menschen infizieren können. Drei davon (SARS-CoV, Middle East Respiratory Syndrome (MERS)CoV und SARS-CoV-2) können eine schwere Infektion der Lunge verursachen. Daten früherer Studien belegen, dass etwa 2 Wochen nach Symptombeginn bei SARSCoV-Infektion bildmorphologisch retikuläre Veränderungen vorliegen, die bei der Hälfte der Patienten nach einem Monat persistieren. Längere Nachbeobachtungsdaten, 15 Jahre später, zeigten, dass bei $4,6 \%$ der mit SARS-CoV infizierten Patienten interstitielle Veränderungen auftraten. Obwohl Patienten, die sich von MERS erholt haben, in der Literatur weniger gut beschrieben sind, gibt es bei etwa einem Drittel der Patienten Hinweise auf fibrotische Anomalien in Röntgenaufnahmen des Thorax [2].

Die mit einer COVID-19-Infektion verbundenen Symptome sind vielfältig und rei- chen in der Akutphase von leichten Symptomen der oberen Atemwege bis hin zu einem schweren Lungenversagen (acute respiratory distress syndrome, ARDS). Dyspnoe und unspezifische thorakale Beschwerden sind 3 bis 6 Monate nach Erkrankung bei etwa 15\% der genesenen Patienten häufig aufgeführte Symptome [3]. Die Post-COVID-19-Lungenparenchymveränderungen können grob in 2 Kategorien eingeteilt werden: die mit einer organisierenden Pneumonie kompatiblen Veränderungen - die bildgebend führend sind und die retikulären Veränderungen, die bis hin zu veritablen Fibrosezeichen wie Traktionsbronchiektasen und Honeycombing reichen $[3,4]$.

Organisierende Pneumonie (OP) ist ein histologisches Muster der Lungenverletzung, das durch die Füllung von Alveolen und Alveolargängen mit spindelförmigen Fibroblasten und Myofibroblasten gekennzeichnet ist, die später Granulationsgewebe bilden. OP ist häufig sekundär eine Folge von Infektionen (einschließlich viralen), Drogenmissbrauch oder Autoimmunerkrankungen und kann in Zusammenhang mit ARDS auftreten. Die typischen Bildgebungsbefunde sind periphere, bilaterale und/oder peribronchovaskuläre Konsolidierungen, insbesondere in den subpleuralen Regionen der Lungenunterlappen mit in gerin- 
gerem Umfang fleckigen Milchglasverdichtungen. Die OP tritt meist bereits während der späten Akutphase der COVID-19-Pneumonie oder in der frühen Rekonvaleszenzphase auf. Bei Post-COVID tritt ein akuter autoinflammatorischer Phänotyp auf: Etwa 4 Wochen nach Genesung sind bildmorphologisch in mehr als der Hälfte der Patienten Milchglasverdichtungen zu sehen. Die Pathologie bei den meisten dieser Patienten entspricht einer lymphozytären Inflammation und organisierender Pneumonie. In einer prospektiven Studie mit COVID19-Patienten 8 Wochen nach Entlassung, hatten 7\% respiratorische Beschwerden und zeigten persistente radiologische Veränderungen, hinweisend auf eine interstitielle Lungenerkrankung (interstitial lung disease, ILD). Bei der Mehrheit dieser Patienten (4\%) wurde eine OP diagnostiziert und diese Patienten konnten klinisch und lungenfunktionell von einer Kortisontherapie profitieren. Mitunter konnte die deutlich aggressivste akute Variante der $\mathrm{OP}$, eine Akute fibrotische organisierende Pneumonie (AFOP) auftreten $[4,5]$.

Viele Überlebende einer schweren COVID19-Infektion weisen in ihren Thorax-CTScans nach der Genesung interstitielle Veränderungen auf, die als «Post-COVID-19 ILD» bezeichnet werden [6]. Die retikulären Veränderungen können entweder einer IPF-typischen Ausbreitung folgen (subpleural, basal) und sind damit mutmaßlich auch Ausdruck einer aggravierten IPF; oder sie können eher peribronchovaskulär und zentraler auftreten, und weisen dann wahrscheinlich tatsächlich auf eine Erschöpfung des lokalen, alveolären Stammzellpools, mit einer nachgeschalteten Bronchialisierung der distalen Lufträume hin. Eine Beobachtungs-Kohortenstudie verwendete CT-Scans und diagnostizierte PostCOVID-19-ILD bei mehr als der Hälfte von 81 Überlebenden einer schweren COVID19-Pneumonie. In einer prospektiven Kohorte von Patienten mit COVID-19-Pneumonie bestanden bei 62\% der Patienten im CT-Thorax 6 Monate nach Entlassung verbleibende CT-Anomalien: Dies umfasste 35\% der Gesamtkohorte mit «fibrotischen» Merkmalen wie retikuläre Veränderungen, Traktionsbronchiektasen und Honigwaben. Der Zusammenhang zwischen ARDS und fibroseähnlichen Veränderungen nach 6 Monaten war besonders auffällig: ARDS war bei 63\% dieser Patienten vorhanden, jedoch nur bei $8 \%$ der Patienten ohne fibrotische Veränderungen. Die serielle CTAuswertung zeigte eine Zunahme der Merkmale, die auf eine zugrunde liegende Fibrose hindeuten, aber ebenso eine Abnahme der Milchglasverdichtungen und Konsolidierungen. Das Ausmaß der fibroseähnlichen Veränderungen war nach 6 Monaten positiv korreliert mit dem Gesamtausmaß der Erkrankung und dem Ausmaß der Milchglasverdichtungen im Aufnahme-CT [7].

Progressive Lungenfibrose kann bei einem erheblichen Anteil der Patienten mit ARDS eine Todesursache sein, während bei einem signifikanten Anteil der Überlebenden eine langfristige Beeinträchtigung der Lungenfunktion und radiologische Auffälligkeiten gezeigt werden, die auf eine Lungenfibrose hinweisen. In einer AutopsieStudie von 30 Patienten, die an COVID-19 verstorben waren, wurde eine histopathologische Progression von diffusen alveolären Schäden (diffuse alveolar damage, DAD) zum fibrosierenden Muster in 43\% der Proben beobachtet [1].

Faktoren, die mit dem Nachweis einer Progression zu COVID-19-ILD in Verbindung gebracht wurden, sind höheres Alter, erhöhte Atemfrequenz, komorbide arterielle Hypertonie und Behandlung auf der Intensivstation. Laborrisikofaktoren für fibrotische Merkmale waren höheres C-reaktives Protein (CRP), niedrige Lymphozytenzahl, niedrigere Ausgangswerte von Interferon-Gamma (IFN-y) und Monozyten-Chemoattraktant Protein 3 (MCP-3). Interessanterweise zeigten sich in genetischen Studien große Profilähnlichkeiten zwischen COVID-19 und idiopathischer Lungenfibrose [1, 8].

Obwohl die mechanische Beatmung (mechanical ventilation, MV) die wichtigste unterstützende Therapie bei ARDS ist, kann sie Lungenverletzungen - die als ventilation-induced lung injury (VILI) bezeichnet werden - verursachen oder bereits vorhandene Schäden verschlimmern. Thromboembolie und Hyperkoagulabilität können an der Pathogenese der Lungenfibrose bei COVID-19-Pneumonie beteiligt sein. Eine immunologische Dysregulation bei Patienten mit COVID-19, auch bekannt als «Zytokinsturm», kann einen wesentlichen Beitrag zur Multiorgandysfunktion leisten. Immunbedingte Schäden tragen zu COVID-assoziiertem ARDS bei. Die Wirkung einer verlängerten Hypoxie auf die Entwicklung einer interstitiellen Lungenfibrose ist nicht spezifisch für COVID-19, aber in der Literatur gut dokumentiert: Hypoxieinduzierbarer Faktor 1-alpha (HIF-1-alpha) ist an der Initiierung und Progression mehrerer Arten von Gewebefibrosen beteiligt. Es ist unklar, ob die Patientenselbstverschuldete Lungenverletzung (patient selfinflicted lung injury, P-SILI), eine Form der Lungenverletzung, die früh bei ARDS auftritt und durch starke Spontanatmungsanstrengungen zu Lungenschäden führen kann, ein Risikofaktor für die Entwicklung einer Lungenfibrose nach COVID-19-Pneumonie sein kann $[1,3,9,10]$.

Es gibt viele offene Fragen bezüglich der Post-COVID-ILD: Entsprechen die Retikulationen in der frühen Konvaleszenzphase einer COVID-19-Pneumonie dem Residuum von ARDS, einer fibrotischen OP oder einer irreversiblen Fibrose? Sind Milchglasverdichtungen Zeichen einer reversiblen Krankheit oder das Vorstadium des Progresses zur Fibrose? Wenn ein typisches UIPMuster auftritt, entspricht dies einer COVID19-Lungenfibrose oder war die COVID19-Infektion der sogenannte ssecond hit bei prädisponierten Patienten zur Entwicklung einer IPF? Wann ist der richtige Zeitpunkt für die invasive Diagnostik, wann ist der richtige Zeitpunkt zur Einleitung einer Kortisontherapie oder einer antifibrotischen Therapie? Um festzustellen, ob es sich bei den radiologischen Retikulationen bei einer COVID-19-Pneumonie um eine irreversible Fibrose handelt, ist definitiv eine Langzeitnachsorge erforderlich, obwohl v.a. ältere Patienten mit schwerer COVID19-Erkrankung anscheinend anfälliger für eine Fibrose sind. Es scheint sinnvoll, bei Patienten mit persistierender Symptomatik den diagnostischen Algorithmus der ILDs zu verwenden - einschließlich der invasiven Diagnostik mittels Bronchoskopie sowie der interdisziplinären Fibrosekonferenz. Bei klinisch, radiologisch und lungenfunktionell progredienter Lungenfibrose kann 
eine Therapie mit dem antifibrotischen Medikament Nintedanib diskutiert werden [11]. Daten aus randomisierten Studien zur Prävalenz und systematischen Therapie von Post-COVID-ILD sind dringend notwendig, um diese Patienten optimal zu behandeln. Zudem können uns <real-life Data〉 aus Post-COVID- und Long-COVID-Ambulanzen und -Studien helfen, den Verlauf der Post-COVID-ILD besser zu verstehen.

\section{Literatur}

1 Ambardar SR, Hightower SL, Huprikar NA, et al.: Post-COVID-19 Pulmonary Fibrosis: Novel Sequelae of the Current Pandemic. J Clin Med. 2021;10(11).

2 Vasarmidi E, Tsitoura E, Spandidos DA, et al.: Pulmonary fibrosis in the aftermath of the COVID-19 era (Review). Exp Ther Med. 2020;20(3):2557-60.

3 Wigen J, Lofdahl A, Bjermer L, et al.: Converging pathways in pulmonary fibrosis and Covid-19 - The fibrotic link to disease severity. Respir Med X. 2020;2:100023.

4 Kory P, Kanne JP: SARS-CoV-2 organising pneumonia: <Has there been a widespread failure to identify and treat this prevalent condition in COVID-19?>. BMJ Open Respir Res. 2020;7(1).
Wir wünschen Ihnen eine anregende Lektüre!

Ihre
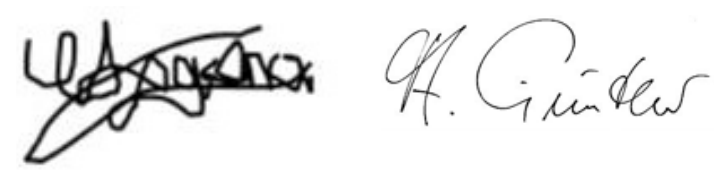

Dr. Fotios Drakopanagiotakis und Prof. Dr. Andreas Günther
5 Myall KJ, Mukherjee B, Castanheira AM, et al.: Persistent Post-COVID-19 Interstitial Lung Disease. An Observational Study of Corticosteroid Treatment. Ann Am Thorac Soc. 2021;18(5):799-806.

6 Athol U, Wells AD, Sujal R: Desai. Interstitial Lung Disease after COVID-19 Infection: A Catalog of Uncertainties 2021. Radiology. 2021;299:1, E216-E218.

7 Han X, Fan Y, Alwalid O, et al.: Six-month Follow-up Chest CT Findings after Severe COVID-19 Pneumonia. Radiology. 2021; 299(1):E177-E86.
8 Aronson KI, Podolanczuk AJ: Lungs after COVID-19:Evolving Knowledge of Post-COVID-19 Interstitial Lung Disease. Ann Am Thorac Soc. 2021;18(5):773-4.

9 Liang Y, Wang ML, Chien CS, et al.: Highlight of Immune Pathogenic Response and Hematopathologic Effect in SARS-CoV, MERS$\mathrm{CoV}$, and SARS-Cov-2 Infection. Front Immunol. 2020;11:1022.

10 Udwadia ZF, Koul PA, Richeldi L: Post-COVID lung fibrosis: The tsunami that will follow the earthquake. Lung India. 2021; 38(Supplement):S41-S7.

11 George PM, Wells AU, Jenkins RG: Pulmonary fibrosis and COVID-19:the potential role for antifibrotic therapy. Lancet Respir Med. 2020;8(8):807-15. 